Article

\title{
Fabrication of Bendable Circuits on a Polydimethylsiloxane (PDMS) Surface by Inkjet Printing Semi-Wrapped Structures
}

\author{
Jiazhen Sun ${ }^{1}$, Jieke Jiang ${ }^{2}$, Bin Bao ${ }^{1}$, Si Wang ${ }^{2}$, Min $\mathrm{He}^{2}$, Xingye Zhang ${ }^{2}$ and Yanlin Song ${ }^{1,2, *}$ \\ 1 School of Chemistry and Environment, Beihang University, Beijing 100191, China; \\ jiazhensun@buaa.edu.cn (J.S.); baobin@iccas.ac.cn (B.B.) \\ 2 Key Laboratory of Green Printing, Institute of Chemistry, Chinese Academy of Sciences, \\ Beijing Engineering Research Center of Nanomaterials for Green Printing Technology, \\ Beijing National Laboratory for Molecular Sciences, Beijing 100190, China; jiangjieke@iccas.ac.cn (J.J.); \\ wangsi@iccas.ac.cn (S.W.); heminyiwen@iccas.ac.cn (M.H.); zhangxy@iccas.ac.cn (X.Z.) \\ * Correspondence: ylsong@iccas.ac.cn; Tel.: +86-10-6252-9284 \\ Academic Editor: Jordi Faraudo \\ Received: 14 February 2016; Accepted: 21 March 2016; Published: 30 March 2016
}

\begin{abstract}
In this work, an effective method was developed to fabricate bendable circuits on a polydimethylsiloxane (PDMS) surface by inkjet printing semi-wrapped structures. It is demonstrated that the precured PDMS liquid film could influence the depositing morphology of coalesced silver precursor inkjet droplets. Accordingly, continuous and uniform lines with a semi-wrapped structure were fabricated on the PDMS surface. When the printed silver precursor was reduced to Ag nanoparticles, the fabricated conductive film exhibited good transparency and high bendability. This work presented a facile way to fabricate flexible patterns on a PDMS surface without any complicated modification or special equipment. Meanwhile, an in situ hydrazine reduction of $\mathrm{Ag}$ has been reported using the vapor phase method in the fabricating process.
\end{abstract}

Keywords: inkjet droplet; precured PDMS; depositing morphology; bendable circuit

\section{Introduction}

Recently, printed electronics have attracted great attention in various device fabricating areas, such as liquid crystal displays, solar cells, organic light emitting diodes, electroluminescent devices and touch screen panels [1-6]. By virtue of its transparency, flexibility and good biocompatibility, polydimethylsiloxane (PDMS) has played an important role in patterning functional materials and fabricating devices, such as transfer printing, microfluidics and implantable medical devices [7-11]. Combining conductive patterns with PDMS will provide a way to fabricate various high-performance devices, such as stretchable conductors, wearable devices and electronic skins [12-16], which require electrodes for control and detection. In previous research, lots of work has been devoted to fabricating conductive patterns on a PDMS surface [17-21], but when using these approaches it is commonly difficult to fabricate flexible circuits on the surface.

Comparing with conventional patterning technologies, such as lithographic printing, nano-imprinting and micro-contact printing [22-27], inkjet printing provides a direct depositing technique using liquid materials, which is low-cost, convenient, flexible and fast. Inkjet printing has been recognized as one of the most promising methods to fabricate patterns on various substrates [28-35]. However, it is still a challenge to inkjet print controllable patterns on a low-adhesive PDMS surface [36-38], because the coalesced inkjet droplets tend to become a larger droplet due to the sliding three phase contact line with a driving force of shape relaxation [39-42]. In the traditional method, there would need to be some modifications to the PDMS surface before inkjet printing, such as 
oxygen plasma treatment or grafting polymer $[43,44]$. However, these methods also could not produce an ideal bendable circuit on the PDMS surface.

In this paper, we reported a facile strategy to fabricate bendable circuits on a PDMS surface by inkjet printing semi-wrapped structures. Trifluoroacetic acid silver salt solution was used as the inkjet printing ink. The depositing morphologies of coalesced silver precursor inkjet droplets were observed on the PDMS precursor substrates with different heating times. The precured PDMS liquid film played a crucial role in adjusting the depositing morphologies of the coalesced inkjet droplets, which was different from the rigid solid substrate. When an inkjet droplet struck the precured PDMS surface, the viscoelasticity of the liquid film could influence the wetting and depositing behavior of the microdroplet [45-48]. Then the continuous and uniform lines with semi-wrapped structure could be fabricated with the appropriate heating time of the PDMS precursor. When the printed deposits were reduced to Ag nanoparticles (AgNPs) by hydrazine hydrate, the fabricated conductive film exhibited good transparency and high bendability. This work presented a facile way to fabricate flexible patterns on PDMS surface without any modification, such as oxygen plasma treatment or grafting polymer. Meanwhile, an in situ hydrazine reduction of $\mathrm{Ag}$ has been reported using the vapor phase method in the inkjet printing process.

\section{Results and Discussion}

\subsection{Fabrication and Characterization of the Inkjet Printed Circuit on PDMS Surface}

Scheme 1 shows the process of fabricating bendable circuit on PDMS surface by inkjet printing. A precured PDMS liquid film was used as the printing substrate. The Ag precursor solution was directly inkjet printed on the substrate. After heating the patterned substrate and reducing the inkjet printed deposits with hydrazine hydrate, the semi-wrapped conductive structures were directly fabricated on the surface of a PDMS film.

Inkjet Printing

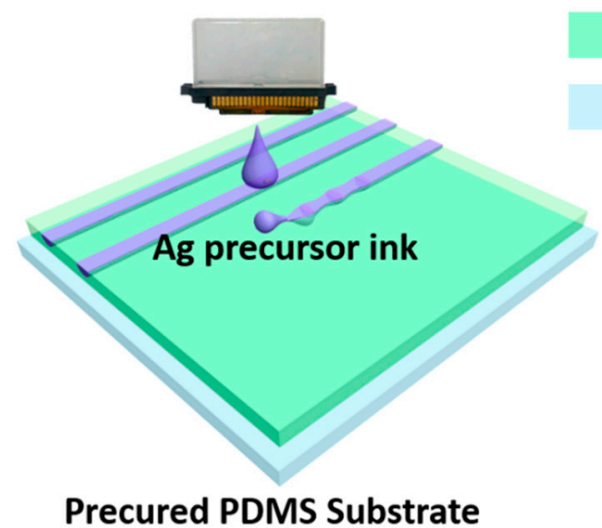

Precured PDMS Substrate
Liquid PDMS

Solidified PDMS

Curing

Reduction

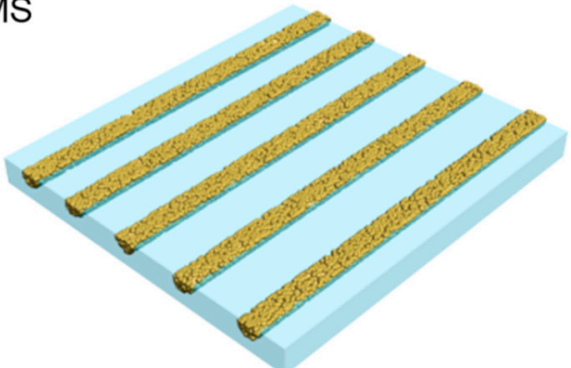

Bendable Conductive Film

Scheme 1. Schematic illustration of the fabricating bendable circuit on a precured PDMS liquid film by inkjet printing.

As shown in Figure 1a, the precise conductive grid was fabricated on the PDMS surface by inkjet printing and in situ vapor phase reduction. Meanwhile, the patterned PDMS film exhibited good transparency with the background. Figure $1 \mathrm{~b}$ demonstrates that the precise grid was fabricated on the PDMS surface, which was composed of continuous and uniform lines. It indicated that this method could provide a route to fabricate circuits on the PDMS surface on a large scale without any complicated process, which will be of great significance for practical application. 


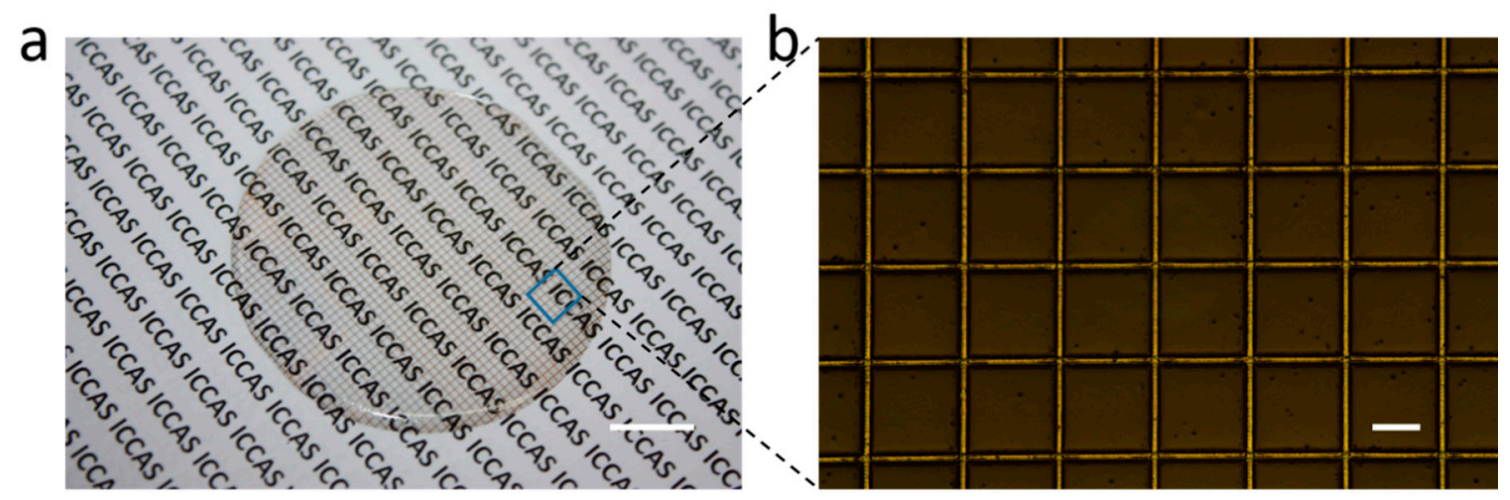

Figure 1. Structure of the inkjet-printed PDMS film. (a) A photograph of the patterned PDMS film, indicating its transparency. Scale bar $1 \mathrm{~cm}$; (b) Magnified optical microscope observation of the inkjet-printed grid on PDMS surface. Scale bar $500 \mu \mathrm{m}$.

\subsection{Depositing Morphologies of Coalesced Inkjet Droplets on the Precured PDMS Liquid Film}

A PDMS surface is a low adhesive surface, so a droplet slips and moves easily on it. Usually a complex pretreatment is required to inkjet print controllable patterns on such a surface, because there will be a force driving shape relaxation of the coalesced droplets when releasing the surface energy. As the viscoelasticity of the precured PDMS liquid film could influence the wetting and depositing behaviors of the microdroplet, the PDMS precursor substrates with a varied heating time were used to adjust the depositing morphologies of coalesced inkjet droplets. The optical images in Figure 2a-d shows that there were two typical depositing morphologies of the coalesced inkjet droplets: uniform line structure and bulged line structure. Figure $2 \mathrm{a}, \mathrm{b}$ shows that short heating time of the PDMS precursor substrate resulted in a uniform line structure, whereas the bulged line structure was generated with relative long heating time for the PDMS precursor substrate (Figure 2c,d). To fabricate uniform lines by inkjet printing, matching surface tension between the liquid substrate and the printing ink is crucial. In our experiment, ethanol as the solvent was used to control the surface tension of ink for matching with the liquid PDMS substrate. In that case, the surface tension of ethanol (about $21.8 \mathrm{mN} / \mathrm{m}$ ) is close to the liquid PDMS substrate (about $21.6 \mathrm{mN} / \mathrm{m}$ ). Uniform lines could thus be inkjet printed on the liquid PDMS film. Meanwhile, the viscoelasticity of the precured PDMS substrate could also contribute to fabricating controllable patterns of the coalescing inkjet droplets. This would provide an opposing force to resist the sliding of the three-phase contact line (TCL) of the inkjet droplet in the shape-relaxing process to fabricate a uniform line [47,49]. By increasing the heating time of the PDMS precursor, the viscoelasticity of the precured PDMS would drop to a low value. Therefore, it could be determined that the heating time of PDMS precursor substrate greatly influenced the depositing morphologies of the coalesced inkjet droplets. As the TCL of the inkjet droplet slides during evaporation on the low-adhesive PDMS surface, it is a difficult task to directly inkjet print on a cured PDMS surface [50].

In order to confirm the location of an inkjet-printed structure on the PDMS substrate, the SEM images of corresponding cross sections were observed, which exhibited the continuous and uniform depositing morphologies in Figure 2a,b. Figure 3a shows the cross section of the printed line with the uncured PDMS substrate. It was observed that the viscous liquid substrate wrapped the inkjet-printed deposits into the PDMS. The printed structure did not exist on the PDMS surface. Figure 3b shows the cross section of printed line on the PDMS precursor substrate after heating for $10 \mathrm{~min}$. It could be observed that the printed structure existed on the PDMS surface, and it could also be partially wrapped into the precured PDMS substrate. Meanwhile, the semi-wrapped structure could contribute to the flexibility of the printed pattern and the gathering of printed materials. Therefore, the appropriate heating time of PDMS precursor substrate could be used to adjust the depositing morphology of inkjet droplets for fabricating continuous and uniform lines with a semi-wrapped structure on the PDMS 
surface. The optical images of printed deposits on the precured PDMS substrate with different focal planes also could indicate the location of an inkjet-printed structure (Figure S1). In the above process, when one droplet impacted the viscoelastic liquid substrate, the ejection of an inkjet droplet provided kinetic energy (Ek) and gravity provided potential energy (Ep). The viscoelastic film would provide a dissipating energy (Ed), which was used to maintain a stable state: $E k+E p=E d$. When the liquid substrate has a short heating time, the high fluidity and low mechanical strength would cause the inkjet droplet to sink into the liquid film. Increasing the heating time, the fluidity of the liquid substrate would decrease and the mechanical strength of the liquid substrate would be enhanced. Once the viscoelasticity of the liquid PDMS film was adjusted to a proper value, the inkjet droplet would deform the surface of the viscoelastic PDMS film due to the impact of the droplet. A local deformation would form at the TCL of inkjet droplet, and the deformed viscoelastic PDMS surface hardly moved as the droplet evaporated. Thus a semi-wrapped structure could be fabricated with the solute precipitated from the inkjet droplet. Meanwhile, the ink concentration determined the shape of the deposit in the microgroove. With the evaporation of inkjet droplet, the density of precipitated silver salt was high enough to settle down with gravity. If the amount of deposit was too little, the deposit would just exist in the bottom of the microgroove. We have shown the printed result with a low ink concentration (3 wt \%) in Figure S2. In our experiment, when the liquid PDMS film was precured with heating for $10 \mathrm{~min}$, the silver salt ink with $15 \%$ mass could fill in the microgrooves.
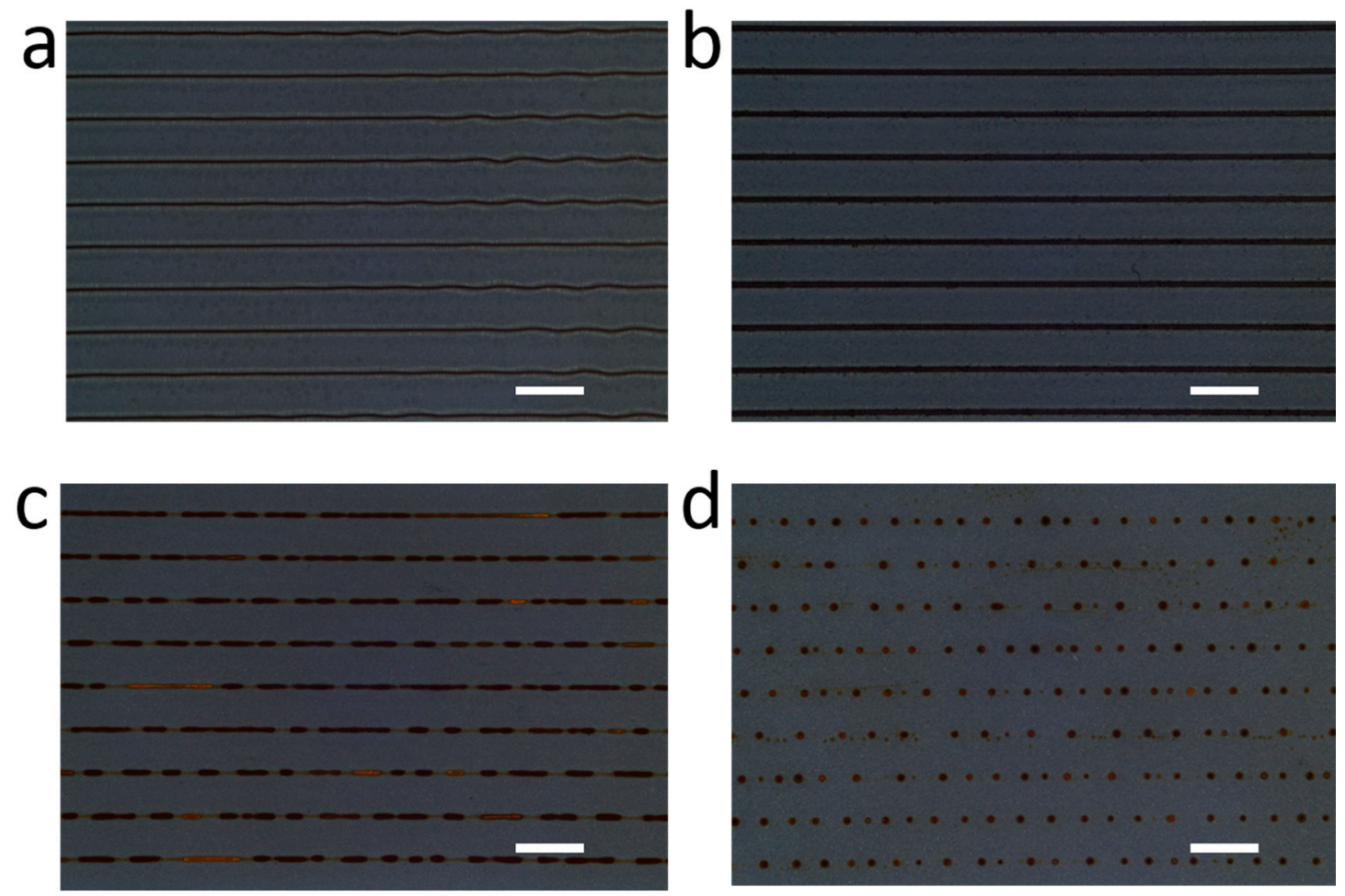

Figure 2. Depositing morphologies of coalesced inkjet droplets with different precured PDMS substrates. (a-d) Optical images of the inkjet printed patterns on the precured PDMS substrates with the heating time at $80{ }^{\circ} \mathrm{C}, 0 \mathrm{~min}, 10 \mathrm{~min}, 20 \mathrm{~min}$ and $30 \mathrm{~min}$, respectively. Scale bar: $500 \mu \mathrm{m}$. When the ink was printed on an uncured PDMS precursor substrate, uniform lines were obtained. With 10 min heating time, the printed ink droplets also could maintain the uniform lines. When the heating time was increased to $20 \mathrm{~min}$, the printed patterns changed to form periodical bulges. When the PDMS precursor substrate was heated for $30 \mathrm{~min}$, the printed patterns tended to form a large discontinuous deposit. 

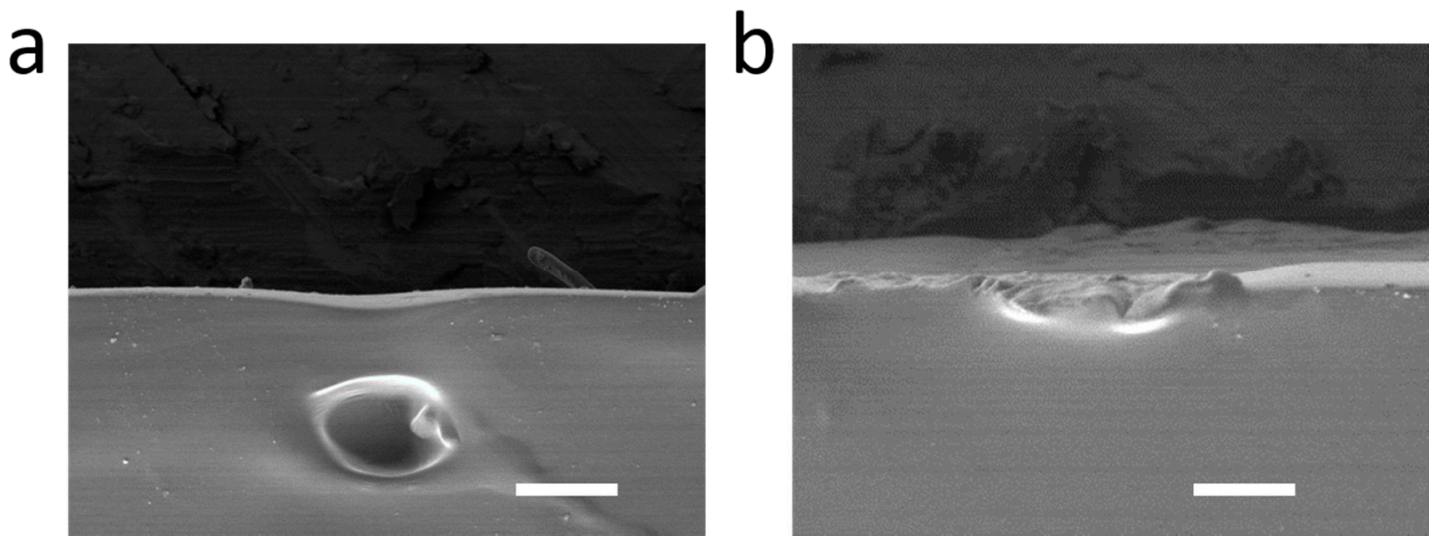

Figure 3. The location of the printed structure on the PDMS surface. (a) Side view SEM image of the printed line corresponding to the optical image in Figure 2a. The deposits were wrapped in the PDMS; (b) Side view SEM image of the printed line corresponding to the optical image in Figure $2 b$. The deposits were semi-wrapped on the PDMS surface. Scale bar: $20 \mu \mathrm{m}$.

\subsection{In Situ Hydrazine Reduction of Ag with Vapor Phase Method}

For printed electronics, metal nanoparticle ink is commonly used due to the high conductivity [51]. The ink consists of metal NPs and a carrier liquid solvent. For practical application, the nanoparticles need specifically designed surface properties which allow them to be stably dispersed in an appropriate solvent [52]. The polymer capping layers would be employed in the formulation of metal nanoparticle inks to prevent particles from aggregation and precipitation. However, the inkjet printing nozzle would be easily clogged with metal nanoparticle ink composed of highly volatile solvents. In this work, we used ethanol as the main solvent to match the surface tension. As ethanol is highly volatile, we used the trifluoroacetic acid silver salt as the solute of ink for good jettability [53]. After the ink was inkjet printed on the substrate, the solvent would evaporate and the Ag precursor was deposited on the substrate. Then the printed PDMS film was placed in a desiccator together with hydrazine hydrate. The desiccator was vacuumized for $2 \mathrm{~min}$, and placed in an $80^{\circ} \mathrm{C}$ oven for two hours. The vapor phase of hydrazine hydrate could provide good contact with the Ag salt in the thermal environment with a low pressure. As shown in Figure 4a-d, the Ag precursor was reduced to AgNPs. Particle size of the AgNPs was about 50-100 nm, which is shown in Figure S3. Comparing to the solution immersion method, this method of vapor phase reduction is a mild way to generate a conductive structure.

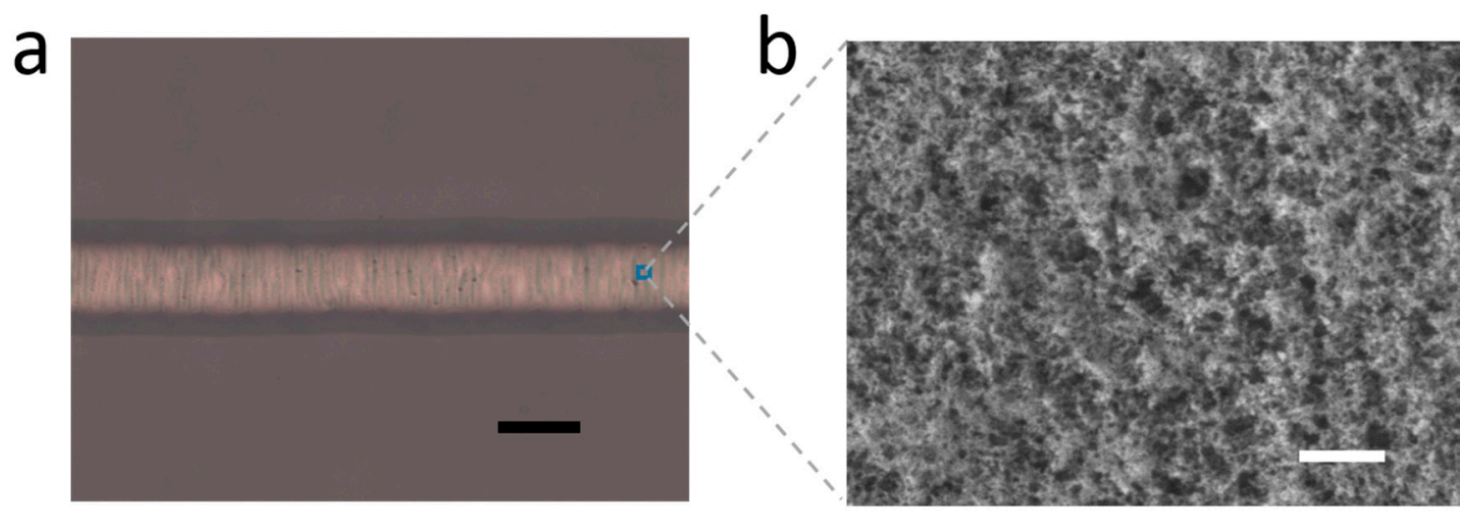

Figure 4. Cont. 

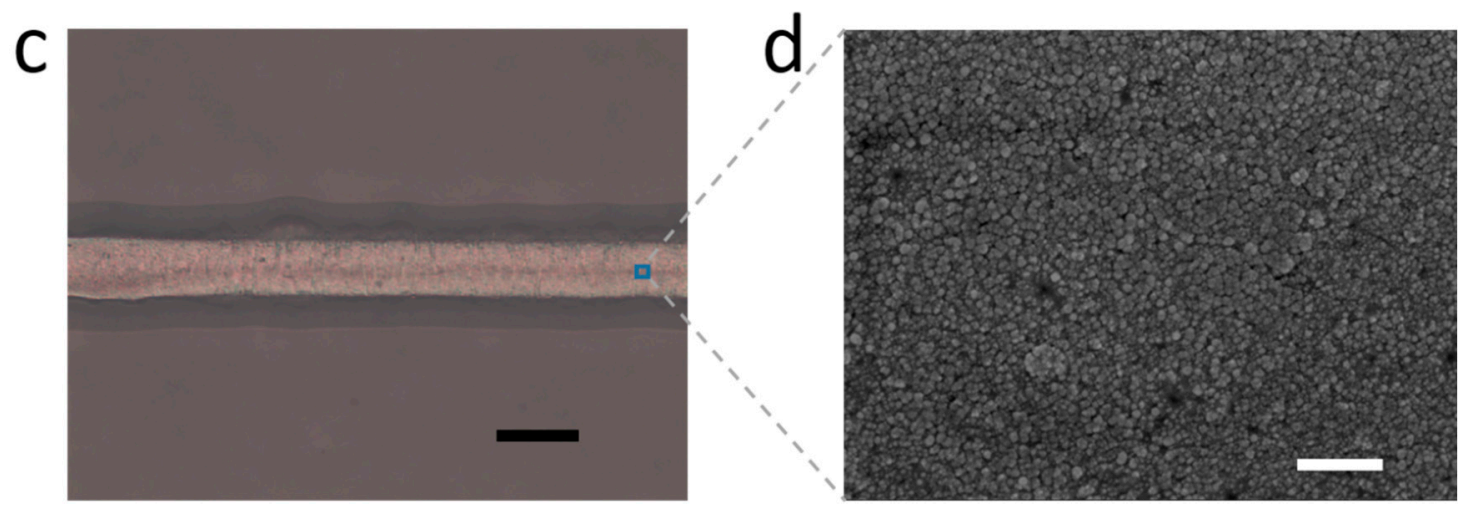

Figure 4. The structural transformation of the printed deposits after reduction with hydrazine hydrate. (a) Optical images of printed deposits before reduction; (b) magnified SEM image of the printed deposits before reduction; (c) Optical images of printed deposits after reduction; (d) magnified SEM image of the printed deposits after reduction, indicative of the AgNPs generation. (a,c: scale bar: $50 \mu \mathrm{m}) ;($ b,d: scale bar: $500 \mathrm{~nm})$.

\subsection{Measurement of Transparency and Conductivity}

Transparency is an important property for the applications of PDMS. As shown in Figure $5 \mathrm{a}$, the transmittance of the printed PDMS film with different grid spacings showed good transparency after reduction in the visible spectra. The inkjet-printed line width was a crucial factor, which would influence the transmittance of the patterned PDMS film. The transmittance could be further improved by decreasing the printed line width while controlling the semi-wrapped structure or using other conductive materials [54]. Furthermore, the resistivity of reduced structure was measured as $2.93 \times 10^{-6} \Omega \cdot \mathrm{m}$ (the average of five independent measurements) by testing the conductivity of a certain segment. The I-V behavior of the printed circuit is shown in Figure 5b. An independent resistance test of the different reduced lines was also carried out, and the Ag lines showed good repeatability of conductivity (Figure S4).
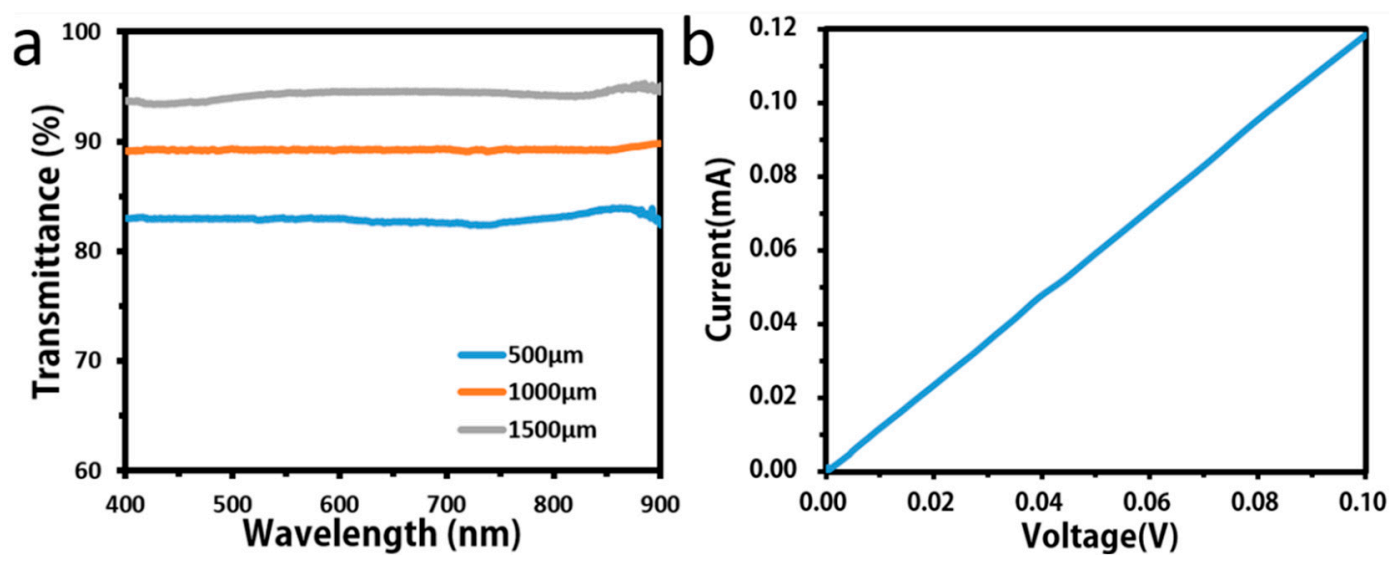

Figure 5. Transmittance spectra and current-voltage curve of the printed PDMS film after reduction. (a) The transmittance of the patterned PDMS with different grid spacings; (b) the printed circuits exhibited linear increase of the current when the sweeping voltage increased, indicating good ohmic contact among AgNPs.

\subsection{Conductive Test of the Bent Semi-Wrapped Circuit}

In this work, the reduced AgNPs were semi-wrapped into the PDMS film. As the semi-wrapped AgNPs were encapsulated by PDMS, the fabricated silver circuits could be used to resist bending 
without apparent resistance increase [55]. As a comparison, circuits inkjet printed on a PET surface using the Ag precursor ink were tested as the control sample. Then the two kinds of printed circuits were bent to $120^{\circ}-150^{\circ}$, and the resistivity of printed circuits before and after the bending was measured by testing the I-V curve of the bent segment. Figure 6a shows that the resistance between the semi-wrapped circuit and circuit on PET film was not significantly different before the bending. When the semi-wrapped circuit was bent to $120^{\circ}-150^{\circ}$, the circuit remained conductive with only a little resistance change between $200 \pm 10 \Omega$. While the circuit on PET film was bent to $120^{\circ}-150^{\circ}$, the resistance increased quickly and could not recover when the PET was flattened out. The stable conductivity of the semi-wrapped circuit resulted from the protection of the encapsulation structure. Then, the resistance after repeated bending was tested, comparing with the resistance of the sample without bending. Figure $6 \mathrm{~b}$ shows that there was no apparent resistance increase after the semi-wrapped circuit was bent 800 times. After the bending, an LED was still lit with the bent film as a conductive unit (Figure S5), which demonstrated the excellent bendability of the fabricated circuits.
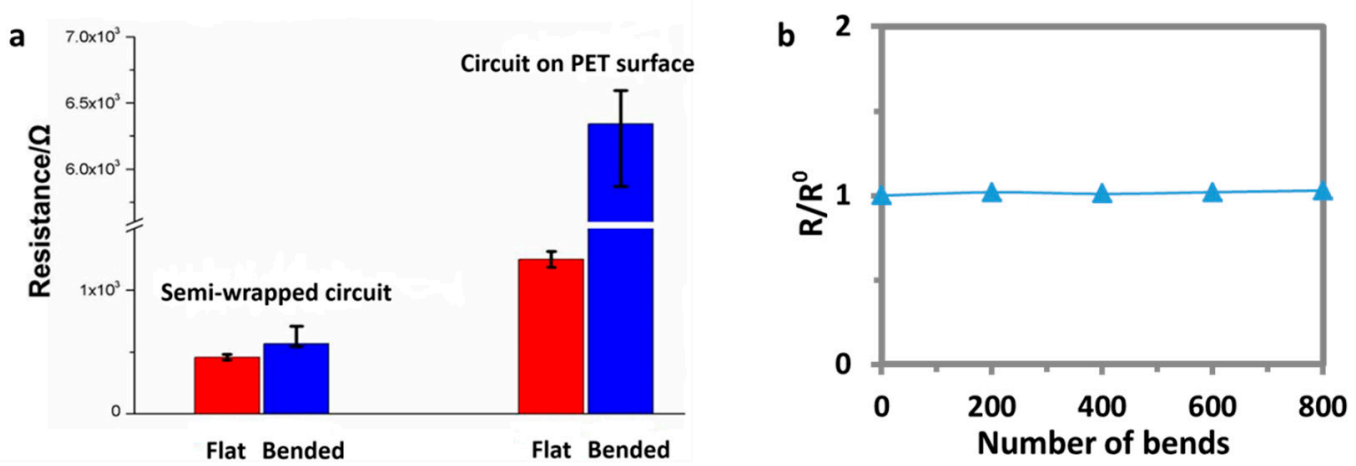

Figure 6. The resistance change of the bent semi-wrapped circuit. (a) The conductive comparison of the semi-wrapped circuit and circuit on PET surface before and after the bending; (b) The resistance change of the semi-wrapped circuit with repeated bending, $R, R^{\circ}$ indicate the resistance of the semi-wrapped circuit before and after the bending, respectively.

\section{Experimental Section}

\subsection{Preparation of Printing Ink}

The trifluoroacetic acid silver salt with 15\% mass percent (Sigma-Aldrich) was dissolved in water/ethanol mixed solvent with volume ratio 10:90 (Sinopharm Chemical Reagent Co. Ltd., Beijing, China) and was used as the printing ink. The viscosity of the ink was $1.409 \mathrm{mPa} \cdot \mathrm{s}$. The surface tension of the ink was $23.8 \mathrm{mN} / \mathrm{m}$. The density of the ink was $0.962 \mathrm{~g} / \mathrm{cm}^{3}$. The solution was injected into an ink cartridge for use as printing ink.

\subsection{Preparation of the Precured PDMS Substrates}

PDMS base was mixed with a curing agent in the proportion of 10:1 by weight to make PDMS precursor (Sylgard 184, Dow Corning, Midland, MI, America). The precursor was put into a centrifuge to remove air bubbles (1000 rpm, 2 mins), then it was put onto a hydrophobic silicon wafer by spin-coating at $800 \mathrm{rpm}$ for $20 \mathrm{~s}$. The height of the liquid PDMS film was about $150 \mu \mathrm{m}$. Subsequently, the PDMS precursor was precured in an $80^{\circ} \mathrm{C}$ oven for a given time and used as the printing substrate.

\subsection{Inkjet Printing Silver Precursor Ink}

The inkjet printing process was performed via a Dimatix Materials Printer (FUJIFILM DMP-2800 series, Tokyo, Japan) with one $25 \mu \mathrm{m}$ nozzle and $10 \mu \mathrm{m}$ inkjet droplet distance controlled with the Dimatix Drop Manager software. The environment temperature of the inkjet printing was 
$25^{\circ} \mathrm{C}$. A single nozzle was used during the printing process. The printing frequency was $5.0 \mathrm{kHz}$. A customized waveform was utilized with piezoelectric maximum $30 \mathrm{~V}$ and pulse width $8.5 \mu \mathrm{s}$.

\subsection{Reducing the Printed Patterns}

The printed patterns was placed in a desiccator together with $25 \mathrm{~mL}$ hydrazine hydrate (Sinopharm Chemical Reagent Co. Ltd., Beijing, China). The desiccator pumped air for 2 min and was then placed in an $80^{\circ} \mathrm{C}$ oven for two hours.

\subsection{Electrical Measurement}

The electrical measurement was characterized under ambient conditions, using a semiconductor system (Keithley 4200-SCS, Keithley Instruments Inc, Cleveland, OH, USA) and an analytical probe station (Suss PM5). Two platinum conical probes were placed to contact the printed pattern, then applied impressed voltage to detect the corresponding current. The cross-sectional area was measured according to the scanning electron microscope (SEM) images. The resistivity $\rho$ was calculated from the resistance $R$, the length $L$, and the cross-sectional area $A$ of the measured conductive segment, using $\rho=R \cdot A / L$.

\subsection{Characterization}

The structures of the printed patterns were investigated using an SEM (JSM-7500, JEOL Ltd, Tokyo, Japan). Optical micrographs were acquired with an optical microscope (Olympus MX40, Olympus Corporation, Tokyo, Japan). The transmittance of the printed PDMS conductive film was measured using a UV-Vis spectrophotometer (Shimadzu, UV 2600, Kyoto, Japan).

\section{Conclusions}

In summary, we demonstrated that a precured PDMS liquid substrate could be used to control the depositing morphologies of coalesced Ag precursor inkjet droplets. Then, semi-wrapped lines were fabricated on the PDMS surface. After the printed deposits were reduced to AgNPs by the in situ vapor phase reduction, a conductive film with good transparency and high bendability was fabricated. This work will be of significance for fabricating flexible circuits and interconnections in printed electronics.

Supplementary Materials: The following are available online at www.mdpi.com/1996-1944/9/4/253/s1.

Acknowledgments: We gratefully acknowledge financial support from the National Natural Science Foundation of China (Nos. 51173190, 51473173, 51573193, 21203209, 21121001, 21303220), the National Basic Research Program of China (973 Program) (No.2013CB933004), and the "Strategic Priority Research Program" of the Chinese Academy of Sciences (No. XDA09020000).

Author Contributions: Yanlin Song and Min He conceived and designed the experiment; Xingye Zhang and Si Wang reviewed the manuscript technically and grammatically; Bin Bao and Jieke Jiang conducted the experiment and analyzed the data; Jiazhen Sun performed the experiments and wrote the manuscript.

Conflicts of Interest: The authors declare no conflict of interest.

\section{References}

1. Layani, M.; Kamyshny, A.; Magdassi, S. Transparent conductors composed of nanomaterials. Nanoscale 2014, 6, 5581-5591. [CrossRef] [PubMed]

2. Li, Y.W.; Mao, L.; Gao, Y.L.; Zhang, P.; Li, C.; Ma, C.Q.; Tu, Y.F.; Cui, Z.; Chen, L.W. ITO-free photovoltaic cell utilizing a high-resolution silver grid current collecting layer. Sol. Energy Mat. Sol. C 2013, 113, 85-89. [CrossRef]

3. Kim, J.; Park, C.J.; Yi, G.; Choi, M.S.; Park, S.K. Low-temperature solution-processed gate dielectrics for high-performance organic thin film transistors. Materials 2015, 8, 6926-6934. [CrossRef] 
4. Hsu, P.; Wang, S.; Wu, H.; Narasimhan, V.K.; Kong, D.S.; Lee, H.R.; Cui, Y. Performance enhancement of metal nanowire transparent conducting electrodes by mesoscale metal wires. Nat. Commun. 2013, 4, 1-7. [CrossRef] [PubMed]

5. Xu, W.Y.; Zhao, J.W.; Qian, L.; Han, X.Y.; Wu, L.Z.; Wu, W.C.; Song, M.S.; Zhou, L.; Su, W.M.; Wang, C.; et al . Sorting of large-diameter semiconducting carbon nanotube and printed flexible driving circuit for organic light emitting diode (OLED). Nanoscale 2014, 6, 1589-1595. [CrossRef] [PubMed]

6. Bao, C.X.; Yang, J.; Gao, H.; Li, F.M.; Yao, Y.F.; Yang, B.; Fu, G.; Zhou, X.X.; Yu, T.; Qin, Y.Q.; et al. In situ fabrication of highly conductive metal nanowire networks with high transmittance from deep-ultraviolet to near-infrared. ACS Nano 2015, 9, 2502-2509. [CrossRef] [PubMed]

7. Kang, S.M.; You, I.; Cho, W.K.; Shon, H.K.; Lee, T.G.; Choi, I.S.; Karp, J.M.; Lee, H. One-step modification of superhydrophobic surfaces by a mussel-inspired polymer coating. Angew. Chem. Int. Ed. 2010, 49, 9401-9404. [CrossRef] [PubMed]

8. Domachuk, P.; Tsioris, K.; Omenetto, F.G.; Kaplan, D.L. Bio-microfluidics: Biomaterials and biomimetic designs. Adv. Mater. 2010, 22, 249-260. [CrossRef] [PubMed]

9. Whitesides, G.M. The origins and the future of microfluidics. Nature 2007, 442, 368-373. [CrossRef] [PubMed]

10. Kim, S.H.; Moon, J.H.; Kim, J.H.; Jeong, S.M.; Lee, S.H. Flexible, stretchable and implantable PDMS encapsulated cable for implantable medical device. Biomed. Eng. Lett. 2011, 1, 199-203. [CrossRef]

11. Hou, J.; Zhang, H.C.; Yang, Q.; Li, M.Z.; Song, Y.L.; Jiang, L. Bio-inspired photonic-crystal microchip for fluorescent ultratrace detection. Angew. Chem. Int. Ed. 2014, 53, 5791-5795. [CrossRef] [PubMed]

12. Larmagnac, A.; Eggenberger, S.; Janossy, H.; Vosro, J. Stretchable electronics based on Ag-PDMS composites. Sci. Rep. 2014, 4, 1-7. [CrossRef] [PubMed]

13. Cheng, T.; Zhang, Y.; Lai, W.Y.; Huang, W. Stretchable thin-film electrodes for flexible electronics with high deformability and stretchability. Adv. Mater. 2015, 27, 3349-3376. [CrossRef] [PubMed]

14. Zhou, T.; Zhang, C.; Han, C.B.; Fan, F.R.; Tang, W.; Wang, Z.L. Woven structured triboelectric nanogenerator for wearable devices. ACS Appl. Mater. Interfaces 2014, 6, 14695-14701. [CrossRef] [PubMed]

15. Yi, P.; Awang, R.A.; Rowe, W.S.T.; Kalantar-zadeh, K.; Khoshmanesh, K. PDMS nanocomposites for heat transfer enhancement in microfluidic platforms. Lab Chip 2014, 14, 3419-3426. [CrossRef] [PubMed]

16. Hammock, M.L.; Chortos, A.; Tee, B.C.; Tok, J.B.; Bao, Z.N. The Evolution of Electronic Skin (E-Skin): A brief history, design considerations, and recent progress. Adv. Mater. 2013, 25, 5997-6038. [CrossRef] [PubMed]

17. Chen, M.T.; Zhang, L.; Duan, S.S.; Jing, S.L.; Jiang, H.; Li, C.Z. Highly stretchable conductors integrated with a conductive carbon nanotube/graphene network and 3D porous poly(dimethylsiloxane). Adv. Funct. Mater. 2014, 24, 7548-7556. [CrossRef]

18. Cong, H.L.; Pan, T.R. Photopatternable conductive PDMS materials for microfabrication. Adv. Funct. Mater. 2008, 18, 1912-1921. [CrossRef]

19. Liu, C.X.; Choi, J.W. Patterning conductive PDMS nanocomposite in an elastomer using microcontact printing. J. Micromech. Microeng. 2009, 19,1-8. [CrossRef]

20. Niu, X.Z.; Peng, S.L.; Liu, L.Y.; Wen, W.J.; Sheng, P. Characterizing and patterning of PDMS-based conducting composites. Adv. Mater. 2007, 19, 2682-2686. [CrossRef]

21. Li, H.; Luo, C.X.; Ji, H.; Ouyang, Q.; Chen, Y. Micro-pressure sensor made of conductive PDMS for microfluidic applications. Microelectron. Eng. 2010, 87, 1266-1269. [CrossRef]

22. Ito, T.; Okazaki, S. Pushing the limits of lithography. Nature 2000, 406, 1027-1031. [CrossRef] [PubMed]

23. Zhang, Y.Q.; Kong, X.Y.; Gao, L.J.; Tian, Y.; Wen, L.P.; Jiang, L. Fabrication of nanochannels. Materials 2015, 8, 6277-6308. [CrossRef]

24. Ahn, S.H.; Guo, L.J. High-speed roll-to-roll nanoimprint lithography on flexible plastic substrates. Adv. Mater. 2008, 20, 2044-2049. [CrossRef]

25. Guo, L.J. Nanoimprint lithography: Methods and material requirements. Adv. Mater. 2007, 19, 495-513. [CrossRef]

26. Yan, X.; Yao, J.M.; Lu, G.A.; Chen, X.; Zhang, K.; Yang, B. Microcontact printing of colloidal crystals. J. Am. Chem. Soc. 2004, 126, 10510-10511. [CrossRef] [PubMed]

27. Chen, J.K.; Chang, C.J. Fabrications and applications of stimulus-responsive polymer films and patterns on surfaces: A review. Materials 2014, 7, 805-875. [CrossRef]

28. Tian, D.L.; Song, Y.L.; Jiang, L. Patterning of controllable surface wettability for printing techniques. Chem. Soc. Rev. 2013, 42, 5184-5209. [CrossRef] [PubMed] 
29. Singh, M.; Haverinen, H.M.; Dhagat, P.; Jabbour, G.E. Inkjet printing-process and its applications. Adv. Mater. 2010, 22, 673-685. [CrossRef] [PubMed]

30. Shimoni, A.; Azoubel, S.; Magdassi, S. Inkjet printing of flexible high-performance carbon nanotube transparent conductive films by "coffee ring effect". Nanoscale 2014, 6, 11084-11089. [CrossRef] [PubMed]

31. Abulikemu, M.; Da'as, E.H.; Haverinen, H.; Cha, D.; Malik, M.A.; Jabbour, G.E. In situ synthesis of self-assembled gold nanoparticles on glass or silicon substrates through reactive inkjet printing. Angew. Chem. Int. Ed. 2014, 53, 420-423. [CrossRef] [PubMed]

32. Li, L.H.; Guo, Y.Z.; Zhang, X.Y.; Song, Y.L. Inkjet-printed highly conductive transparent patterns with water based Ag-doped graphene. J. Mater. Chem. A 2014, 2, 19095-19101. [CrossRef]

33. Chen, S.P.; Chiu, H.L.; Wang, P.H.; Liao, Y.C. Inkjet printed conductive tracks for printed electronics. ECS J. Solid State Sci. Technol. 2015, 4, 3026-3033. [CrossRef]

34. Sun, J.Z.; Kuang, M.X.; Song, Y.L. Control and application of "coffee ring" effect in inkjet printing. Prog. Chem. 2015, 27, 929-985.

35. Sun, J.Z.; Bao, B.; He, M.; Zhou, H.H.; Song, Y.L. Recent advances in controlling the depositing morphologies of inkjet droplets. ACS Appl. Mater. Interfaces 2015, 7, 28086-28099. [CrossRef] [PubMed]

36. Wong, T.S.; Kang, S.H.; Tang, S.K.Y.; Smythe, E.J.; Hatton, B.D.; Grinthal, A.; Aizenberg, J. Bioinspired self-repairing slippery surfaces with pressure-stable omniphobicity. Nature 2011, 477, 443-447. [CrossRef] [PubMed]

37. Yao, X.; Hu, Y.H.; Grinthal, A.; Wong, T.S.; Mahadevan, L.; Aizenberg, J. Adaptive fluid-infused porous films with tunable transparency and wettability. Nat. Mater. 2013, 12, 529-534. [CrossRef] [PubMed]

38. Wang, L.B.; Li, F.Y.; Kuang, M.X.; Gao, M.; Wang, J.X.; Huang, Y.; Jiang, L.; Song, Y.L. Interface manipulation for printing three-dimensional microstructures under magnetic guiding. Small 2015, 11, 1900-1904. [CrossRef] [PubMed]

39. Kuang, M.X.; Wang, L.B.; Song, Y.L. Controllable printing droplets for high-resolution patterns. Adv. Mater. 2014, 26, 6950-6958. [CrossRef] [PubMed]

40. He, M.; Zhang, Q.L.; Zeng, X.P.; Cui, D.P.; Chen, J.; Li, H.L.; Wang, J.J.; Song, Y.L. Hierarchical porous surface for efficiently controlling microdroplets' self-removal. Adv. Mater. 2013, 25, 2291-2295. [CrossRef] [PubMed]

41. Van Osch, T.H.J.; Perelaer, J.; de Laat, A.W.M.; Schubert, U.S. Inkjet printing of narrow conductive tracks on untreated polymeric substrates. Adv. Mater. 2008, 20, 343-345. [CrossRef]

42. Liu, M.J.; Wang, J.X.; He, M.; Wang, L.B.; Li, F.Y.; Jiang, L.; Song, Y.L. Inkjet printing controllable footprint lines by regulating the dynamic wettability of coalescing ink droplets. ACS Appl. Mater. Interfaces 2014, 6, 13344-13348. [CrossRef] [PubMed]

43. Chiolerio, A.; Rivolo, P.; Porro, S.; Stassi, S.; Ricciardi, S.; Mandracci, P.; Canavese, G.; Bejtka, K.; Pirri, C.F. Inkjet-printed PEDOT: PSS electrodes on plasma-modified PDMS nanocomposites: Quantifying plasma treatment hardness. RSC Adv. 2014, 4, 51477-51485. [CrossRef]

44. Wu, J.W.; Wang, R.D.; Yu, H.X.; Li, G.J.; Xu, K.X.; Tien, N.C.; Roberts, R.C.; Li, D.C. Inkjet-printed microelectrodes on PDMS as biosensors for functionalized microfluidic systems. Lab Chip 2015, 15, 690-695. [CrossRef] [PubMed]

45. Alizadeh, A.; Bahadur, V.; Shang, W.; Zhu, Y.; Buckley, D.; Dhinojwala, A.; Sohal, M. Influence of substrate elasticity on droplet impact dynamics. Langmuir 2013, 29, 4520-4524. [CrossRef] [PubMed]

46. Park, S.J.; Weon, B.M.; Lee, J.S.; Lee, J.; Kim, J.; Je, J.H. Visualization of asymmetric wetting ridges on soft solids with X-ray microscopy. Nat. Commun. 2014, 5, 1-7. [CrossRef] [PubMed]

47. Bao, B.; Jiang, J.K.; Li, F.Y.; Zhang, P.C.; Chen, S.R.; Yang, Q.; Wang, S.T.; Su, B.; Jiang, L.; Song, Y.L. Fabrication of patterned concave microstructures by inkjet imprinting. Adv. Funct. Mater. 2015, 25, 3286-3294. [CrossRef]

48. Guo, Y.Z.; Li, L.H.; Li, F.Y.; Zhou, H.H.; Song, Y.L. Inkjet print microchannels based on a liquid template. Lab Chip 2015, 15, 1759-1764. [CrossRef] [PubMed]

49. Cira1, N.J.; Benusiglio, A.; Prakash, M. Vapour-mediated sensing and motility in two-component droplets. Nature 2015, 519, 446-450. [CrossRef] [PubMed]

50. Kuang, M.X.; Wang, J.X.; Bao, B.; Li, F.Y.; Wang, L.B.; Jiang, L.; Song, Y.L. Inkjet printing patterned photonic crystal domes for wide viewing-angle displays by controlling the sliding three phase contact Line. Adv. Opt. Mater. 2014, 2, 34-38. [CrossRef] 
51. Zhang, Z.L.; Zhang, X.Y.; Xin, Z.Q.; Deng, M.M.; Wen, Y.Q.; Song, Y.L. Synthesis of monodisperse silver nanoparticles for ink-jet printed flexible electronics. Nanotechnology 2011, 22, 425601-425608. [CrossRef] [PubMed]

52. Gamerith, S.; Klug, A.; Scheiber, H.; Scherf, U.; Moderegger, E.; List, E.J.W. Direct ink-jet printing of Ag-Cu nanoparticle and Ag-precursor based electrodes for OFET applications. Adv. Funct. Mater. 2007, 17, 3111-3118. [CrossRef]

53. Kamyshny, A.; Magdassi, S. Conductive nanomaterials for printed electronics. Small 2014, 15, 3515-3535. [CrossRef]

54. Hong, S.; Yeo, J.; Kim, G.; Kim, D.; Lee, H.; Kwon, J.; Lee, H.; Lee, P.; Ko, S.H. Nonvacuum, maskless fabrication of a flexible metal grid transparent conductor by low-temperature selective laser sintering of nanoparticle ink. ACS Nano 2013, 7, 5024-5031. [CrossRef] [PubMed]

55. Jiang, J.K.; Bao, B.; Li, M.Z.; Sun, J.Z.; Zhang, C.; Li, Y.; Li, F.Y.; Yao, X.; Song, Y.L. Fabrication of transparent multilayer circuits by inkjet printing. Adv. Mater. 2016, 28, 1420-1426. [CrossRef] [PubMed]

(C) 2016 by the authors; licensee MDPI, Basel, Switzerland. This article is an open access article distributed under the terms and conditions of the Creative Commons by Attribution (CC-BY) license (http://creativecommons.org/licenses/by/4.0/). 